\title{
Some Brief Thoughts on the Past and Future of Human-Robot Interaction
}

\author{
KERSTIN DAUTENHAHN, University of Hertfordshire
}

CCS Concepts: • Human-centered computing $\rightarrow$ HCI design and evaluation methods; Human computer interaction $(\mathrm{HCI})$; • Computer systems organization $\rightarrow$ Robotics;

Additional Key Words and Phrases: Human-robot interaction

ACM Reference format:

Kerstin Dautenhahn. 2018. Some Brief Thoughts on the Past and Future of Human-Robot Interaction. ACM Trans. Hum.-Robot Interact. 7, 1, Article 4 (May 2018), 3 pages.

https://doi.org/10.1145/3209769

It is a great honor to write the editorial for the inaugural issue of ACM Transactions of HumanRobot Interaction. This is an exciting time for the HRI community. The journal is a descendant of the fournal of Human-Robot Interaction ( $(H R I)$, which has been successful for many years. Under the umbrella of ACM Transactions, I am sure it will go from strength to strength.

What I am Excited About for HRI. There have been a lot of significant changes in the HRI community over the past 20 years. These include the formation of the highly selective and respected annual ACM/IEEE International Conference on Human-Robot Interaction, which is attracting an ever growing audience and was followed by the provision of a dedicated outlet for HRI research with the formation of the fournal of Human-Robot Interaction. I have also seen the impact of discussions on how research in such an interdisciplinary area can be reviewed fairly. While this still remains a main challenge, the formation of different themes of submissions to the conference in terms of user studies, technical work, HRI design and theory, and methods is the right direction forward. Years ago, I was told by several active researchers that there seems to be only one "recipe" for how to get accepted by the HRI conference; thus, the current realization that different types of work exist that need to be evaluated differently is a major advance. I have also observed extensive discussions on reviewing methods as such, which is not only highly valuable for HRI but also other communities and conferences since, naturally, HRI researchers are attending and being involved in the organization of a range of conferences and journals. The impact of those discussions, therefore, goes beyond the HRI community.

What I Am Worried About for HRI. From its initial baby steps, HRIhas been struggling with the notion of how HRI research should be conducted, analyzed and written up. This is not a surprise since, as I have argued myself many times, human-robot interaction is very different

Author's address: K. Dautenhahn, School of Computer Science, University of Hertfordshire, College Lane, Hatfield, Herts, AL10 9AB, United Kingdom.

Permission to make digital or hard copies of part or all of this work for personal or classroom use is granted without fee provided that copies are not made or distributed for profit or commercial advantage and that copies bear this notice and the full citation on the first page. Copyrights for third-party components of this work must be honored. For all other uses, contact the Owner/Author.

2018 Copyright is held by the owner/author(s).

2573-9522/2018/05-ART4

https://doi.org/10.1145/3209769 
from human-human interaction, human-computer interaction, and human-animal interaction. It is also clearly different from traditional robotics and engineering research. Studying interactions of people, for example, with a mobile manipulator or a humanoid robot, is different from studying which interface and interaction design people prefer on their computer or mobile phone screens, how people interact with their dogs, or how they would want to operate a photocopying machine. Moreover, the concept of "robot" is a moving target - what we considered to be robots 20 years ago is now outdated to a large extent. We have a variety of voice-activated smart speakers now on the market that, for all intents and purposes, might be called robots, and I do not even dare to predict what we might call robots in 50 years time. Robot designs change rapidly, models become obsolete, new robotics companies arise, and other companies may stop trading. Thus we, as a community, do not have a common reference point when we refer to "robot," not even a sample of widely agreed-upon reference points. Of course, we can use the classifications android, humanoid, mechanoid, machine-like, zoomorphic, anthropomorphic, appearance-constrained, and the like, but even within such categories the range of design and interactions we can find varies immensely. Thus, the fact that we have been struggling to define "how to do HRI" did not come as a surprise. It comes with the territory of working toward future and emerging technologies, which are healthy struggles for our community.

What worries me most is that experimental psychology has often emerged as a gold standard of doing empirical HRI work, with well-defined experimental designs, well-defined (often limited) stimuli that participants are presented with, and rigorous methods of statistical analysis (which requires sufficiently large numbers of participants). Due to the constraints of the experimental approach, such work often uses pictures or short videos of robots or human-robot interactions. Increasingly, crowdsourcing methods are used in order to secure a sufficient number of participants and, in general, this work focuses on very precise and specific research questions. What such work cannot address is how real people, in real-world environments, would interact face to face with a real robot, in particular in contexts in which this robot is a complex, intelligent, and multitasking system, with not necessarily entirely predictable behavior let alone a system that will learn and adapt during the interactions. A stimulus (e.g., the behavior of a learning robot) that changes during the experiment does not lend itself well to the paradigm of HRI studies inspired by experimental psychology.

Moreover, while short-term interactions are suitable for scenarios in which people might encounter a robot briefly and only once or a few times (e.g., a receptionist robot in a hotel), in increasing number of studies dealing with scenarios of robot companions, co-workers, and assistants in our daily lives are pointing out the need to study long-term interactions in ecologically valid environments. Studying such complex scenarios for long-term and face-to-face interactions is far, far more time-consuming, research-intensive, and complex to design and execute than the abovementioned studies. They also often do not lend themselves to a strictly quantitative approach and, if working with research prototypes rather than a number of commercially available prototypes, are usually confined to a smaller number of participants for practical purposes. Such work then relies heavily not only on questionnaires but also behavioral measures and qualitative approaches to study immersion, engagement, acceptability, utility, and other issues. Personally, I would like to see more studies with live human-robot interaction, with complex, autonomous robots interacting with people in ecologically valid environments. Such work is not easy, but we cannot shy away from it if we want our HRI results to have implications and social impact outside our labs.

Why I Am Optimistic for HRI. Human-robot interaction is a highly multidisciplinary field-it is a joy to attend the annual conference hearing people's thoughts from a variety of backgrounds and a diversity of opinions. As long as we keep the discussions alive on what HRI research is and what 
the best ways are of "how to do it," we will keep learning and evolving as a research field. We do not need set-in-stone paradigms; we need an open mind to adopt whatever methodology is appropriate for the particular research questions we have in mind. We can adopt methodologies not only from experimental psychology but also from many fields, such as anthropology, ethology, and others. Any of these fields are proper, as far as I am concerned, if we use them wisely and appropriately.

Let us learn more about how people interact with autonomous and intelligent robots and how we can shape the relationship between artificial intelligence and HRI. Let us continue the conversation on how we want the field to evolve.

Received March 2018; accepted April 2018 